\title{
Performance Comparison of Digitized Quaternion Modulation with other Modulation Schemes
}

\author{
Anam Zahra ${ }^{1}$, Qasim Umar Khan ${ }^{1, *}$ \\ ${ }^{1}$ Department of Electrical Engineering, College of Electrical and Mechanical Engineering, NUST, Islamabad, Pakistan
}

\begin{abstract}
In wireless networks signal's security from noise has been a very challenging issue, primarily because of the broadcast nature of communication. This paper focuses on digitized Quaternion Modulation (QM) which gives better performance as compared to QPSK, QAM and QFSK. We compare the performance of quaternion modulation with other modulation schemes in terms of BER using idealistic Additive White Gaussian Noise AWGN channel. This scheme can be used in applications such as Global Positioning System (GPS), satellite and space communication system to reduce errors. The simulation results show superior performance of the proposed digitized Quaternion Modulation over its counterparts. Thus one may trade off bandwidth for
\end{abstract}

\section{Introduction}

The selection of digital modulation technique depends on numerous standards such as bit error rate, power and bandwidth efficiency. To select the most suitable modulation, the criteria depends on the nature of particular application, as one application may require higher accuracy in data reception, whereas the other application requirement could be power or available bandwidth [1].

The quality of service delivered by wireless communication system can be significantly upgraded with the help of appropriate selection of modulation scheme. With the increasing use of wireless communication systems, the demand to keep the information secured from noise has also been increased. Wireless communication and wired communication are quite different and offers numerous compensations, i.e. mobility, low cost, superior productivity, scalability and easy installation capability. In wireless communication, the most prominent limitations \& shortcomings are because of reflection, diffraction and scattering which causes the transmitted signals reach at receiver with different power and time delays. In addition the Bit Error Rate (BER) of wireless medium is comparatively high. These disadvantages introduce destructive effects on data transmission performance in wireless communication. Consequently, error control is essential in wireless applications.

The performance criterion in digital data transmission is determined by Bit Error Rate (BER) which is basically: Number of erroneous bits / Total number of bits. Noise intrudes the transmitted signal \& corrupts the data. The relationship between signal and noise is defined by Signal to Noise Ratio (SNR) which is basically; signal power / noise power. SNR (Signal to
Noise Ratio) is inversely proportional to BER (Bit Error Rate), which shows lesser the BER is the higher the SNR (Signal to Noise Ratio) which results in better communication quality [2].

In wireless communication system forward error correction (FEC) codes are used to reduce the high error rate in transmission [3]. Ever more sophisticated coding and decoding schemes have been used throughout the years to improve the bit error rate. In wireless communication MMSE and ZFE equalizers are used to improve the bit error rate (BER) performance [4]. This paper focuses on a recently Digitized Quaternion Modulation scheme which maintains high level of robustness against errors. Digitized Quaternion Modulation scheme is used to improve the bit error rate performance and achieve reliable communication over a noisy channel.

The rest of the paper is organized as follows: In Section II unit quaternion is discussed. In Section III the system model of digitized Quaternion Modulation is presented. Simulations are discussed in Sections IV followed by conclusion in Section V.

\section{Unit Quaternion}

A complex number, is defined by $a+b i$, where $a$ $\& b$ are real numbers and $i$ is an imaginary number such that $i^{2}=-1$ [5]. Complex numbers are two dimensional vectors space over the real numbers. In addition to $i$, quaternions are constructed by adding two new imaginary units $j$ and $k$ with one real part. A quaternion is an extension of the complex number system [6]. A Quaternion is four dimensional hyper-complex number first discovered by Irish mathematician William Rowan Hamilton in 1843 [7]. Quaternions have been considered of pure theoretical interest for a long time. It is only in

Corresponding author: qasimumar.khan@,ceme.nust.edu.pk 
the last decade that quaternion based algorithms were proposed in signal processing [8].

A quaternion is composed of four components, one real and three imaginary components. It is generally represented in the Cartesian form as:

$$
Q=q_{1}+q_{2} i+q_{3} j+q_{4} k
$$

Where $q_{1}, q_{2}, q_{3} \& q_{4}$ are real numbers, while $i, j$, and $k$ are the standard orthonormal basis for $\mathrm{R}^{3}$.

$$
\begin{aligned}
& i=(1,0,0) \\
& j=(0,1,0) \\
& k=(0,0,1)
\end{aligned}
$$

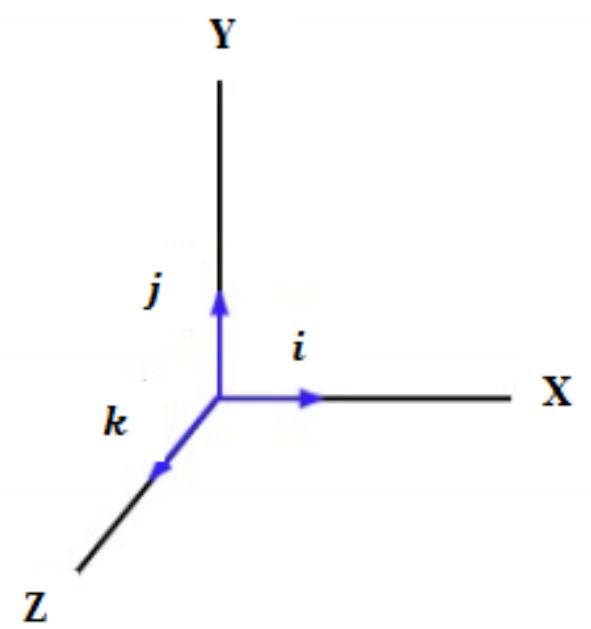

Fig. 1. $i, j, k$ axis

The orthonormal basis components $\mathrm{i}, \mathrm{j}, \mathrm{k}$ [5] as defined in Figure 1 satisfy the following well-known rules introduced by Hamilton;

$$
\begin{gathered}
i^{2}=j^{2}=k^{2}=i j k=-1 \\
i j=k=-j i \\
j k=i=-k j \\
k i=j=-i k
\end{gathered}
$$

This is clearly an extension of complex numbers, where the complex numbers are those quaternions that have $q_{3}=q_{4}=0$ and the real numbers are those quaternions that have $q_{2}=q_{3}=q_{4}=0$ [9]. Basics about hyper-complex numbers systems can be found in [10], while a thorough review about quaternions can be found in [11].

Several properties of complex numbers are extended to quaternions [9].Some of them are;

Conjugation: The conjugate of $Q=q_{1}+q_{2} i+q_{3} j+q_{4} k$, denoted by $\boldsymbol{Q}$ is;

$$
\boldsymbol{Q}=q_{1}-q_{2} i-q_{3} j-q_{4} k
$$

Addition: The sum of two quaternions is;

$$
\begin{gathered}
P \pm Q=\left(p_{1}, p_{2}, p_{3}, p_{4}\right) \pm\left(q_{1}, q_{2}, q_{3}, q_{4}\right) \\
=\left(p_{1} \pm q_{1}, p_{2} \pm q_{2}, p_{3} \pm q_{3}, p_{4} \pm q_{4}\right) \\
=\left(p_{1} \pm q_{1}\right)+\left(p_{2} \pm q_{2}\right) i+\left(p_{3} \pm q_{3}\right) j+\left(p_{4} \pm q_{4}\right) k
\end{gathered}
$$

Multiplication: Multiplication of quaternions by a scalar is the same as complex numbers.

$$
\begin{aligned}
& c Q=c\left(q_{1}, q_{2}, q_{3}, q_{4}\right) \\
& =\left(c q_{1}, c q_{2}, c q_{3}, c q_{4}\right) .
\end{aligned}
$$

Modulus: The modulus of quaternion is represented by $|Q|$ and it can be computed as;

$$
|Q|=\left[q_{1}^{2}+q_{2}^{2}+q_{3}^{2}+q_{4}^{2}\right]^{1 / 2}
$$

Inverse: The inverse of quaternion is denoted by $Q^{-1}$ and it can be computed as;

$$
Q^{-1}=\boldsymbol{Q} /|Q|^{2}
$$

Null Quaternion: A quaternion is said to be null if;

$$
q_{1}=q_{2}=q_{3}=q_{4}=0 .
$$

Non-Commutative Division: The set of quaternions forms non-commutative division algebra. For two quaternions $P \& Q$ non-commutative division algebra can be defined as;

$$
P Q \neq Q P
$$

Unit Quaternion: Conjugate and modulus of quaternions can be used to define unit quaternion. A quaternion is called a unit quaternion (also called a normalized quaternion) if its norm is 1.

$$
|Q|=q_{1}^{2}+q_{2}^{2}+q_{3}^{2}+q_{4}^{2}=1
$$

To obtain a unit quaternion, any quaternion can be normalized by dividing it by its norm. If we donate a unit quaternion as $Q_{u}$, then

$$
Q_{u}=Q /|Q|, \quad|Q| \neq 0
$$

The set of unit quaternions forms a unit sphere in fourdimensional space. Block diagram and detailed system model of digitized Quaternion Modulation scheme is described in next section.

\section{System Model}

Transmitter: One of the most important reasons for choosing Quaternion modulation scheme is to have better performance [12]. Quaternion modulation scheme contains M-FSK (Frequency Shift Keying) modulators. In $\mathrm{M}$-ary signalling the processor considers $\mathrm{k}$ bits at a time; it instructs the modulator to produce one of $M=2^{k}$ waveforms, with $\mathrm{k}=2$ and $\mathrm{M}=4$. FSK modulators are used to generate the basic signals with quadrature carriers illustrated in Figures $2 \mathrm{a} \& 2 \mathrm{~b}$ using carrier frequency $w 1$. The outputs of four FSK systems are $\cos \left(\lambda_{1}(\mathrm{t})\right), \sin \left(\lambda_{2}(\mathrm{t})\right), \cos \left(\delta_{1}(\mathrm{t})\right) \& \sin \left(\delta_{2}(\mathrm{t})\right)$ respectively. 


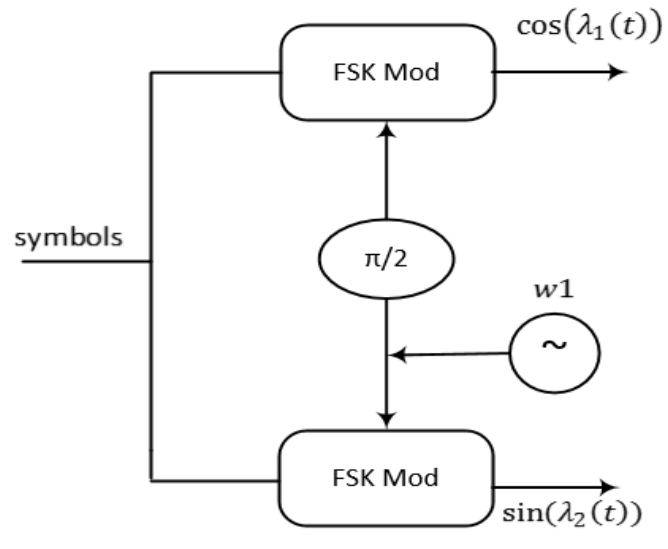

(a)

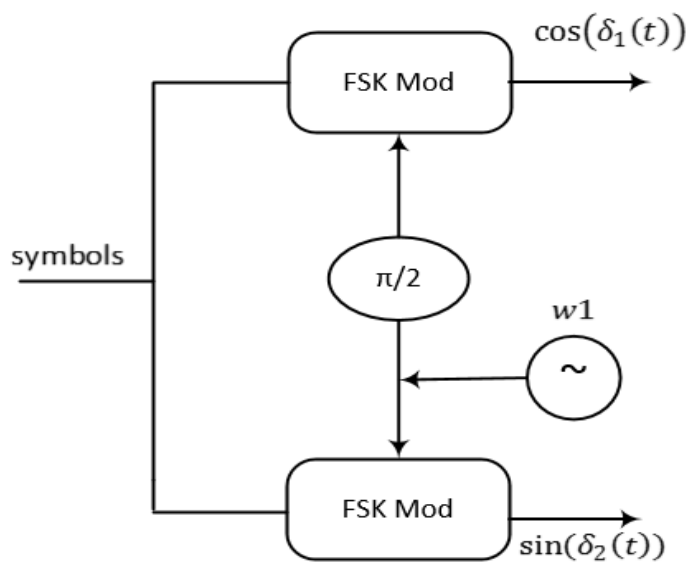

(b)

Fig. 2. (a), (b) Block Diagram for Generating Digitized Quaternion Coordinates

In Figure 3, the digital quaternion coordinates $\mathrm{q}_{1}(\mathrm{t}), \mathrm{q}_{2}(\mathrm{t})$, $\mathrm{q}_{3}(\mathrm{t}) \& \mathrm{q}_{4}(\mathrm{t})$ are computed by combining the signals generated in Figure 2 by FSK modulation. These Quaternion coordinates can be expressed as;

$$
\begin{aligned}
& q_{1}(t)=\cos \left(\lambda_{1}(t)\right) * \sin \left(\delta_{2}(t)\right) \\
& q_{2}(t)=\sin \left(\lambda_{2}(t)\right) * \cos \left(\delta_{1}(t)\right) \\
& q_{3}(t)=\sin \left(\lambda_{2}(t)\right) * \sin \left(\delta_{2}(t)\right) \\
& q_{4}(t)=\cos \left(\lambda_{1}(t)\right) * \cos \left(\delta_{1}(t)\right)
\end{aligned}
$$

Figure 3 shows the block diagram of generation and transmission of quaternion coordinates. To transmit these digitized quaternion coordinates in phase multiplexing and in quadrature multiplexing is done. In multiplexing two high frequency carriers $f_{1} \& f_{2}$ are required to transmit these quaternion co-ordinates. The output from each MUX is then added together to get a final transmitted signal.

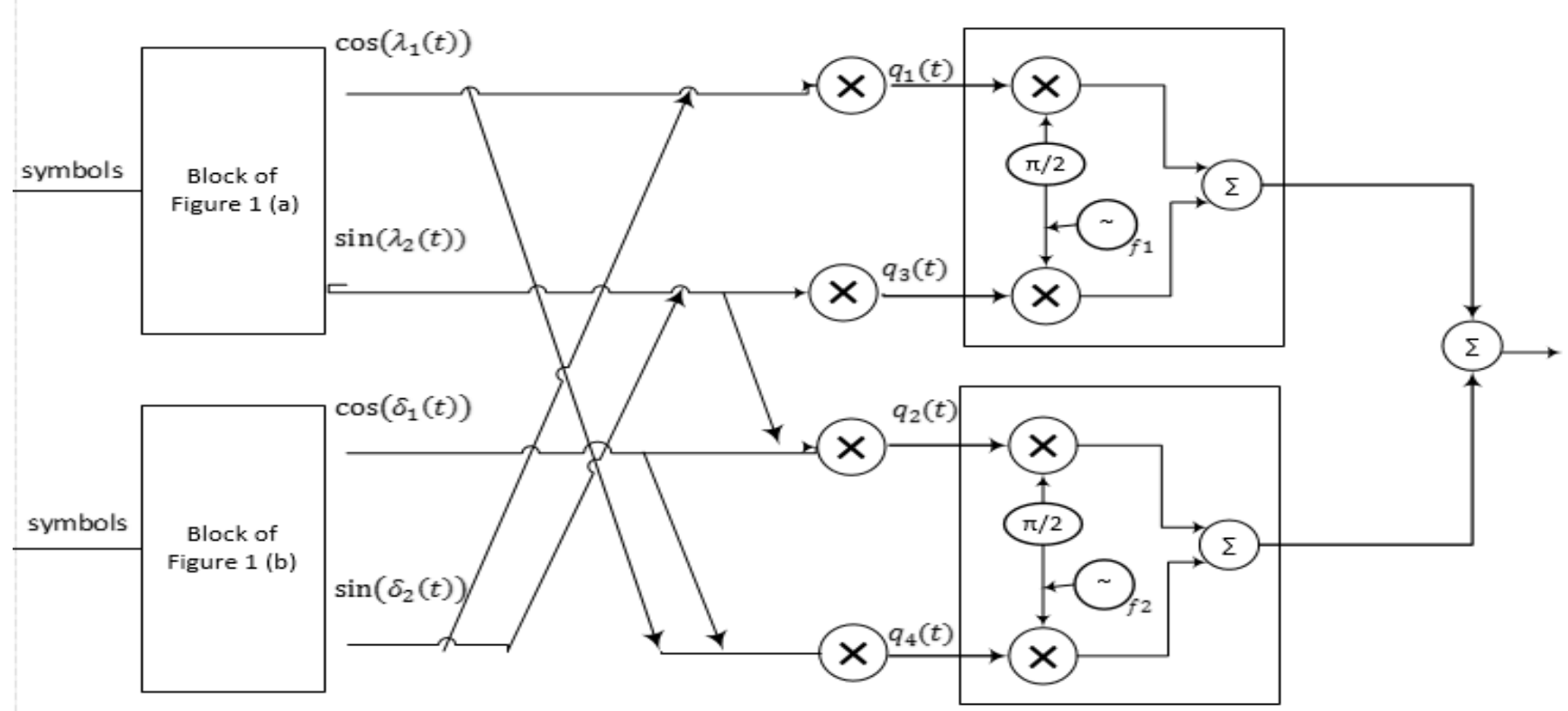

Fig. 3. Two-carrier Digitized Quaternion Modulator

Receiver: The original data is recovered from the modulated signal through Quaternion demodulation technique. In digitized Quaternion demodulation process carrier demodulation is done first and the low pass filter is used to retrieve the quaternion co-ordinates $\mathrm{q}_{1}(\mathrm{t}), \mathrm{q}_{2}(\mathrm{t})$, $\mathrm{q}_{3}(\mathrm{t}) \& \mathrm{q}_{4}(\mathrm{t})$. Squaring operation is performed to prevent the appearance of any negative values.
The outputs $q_{1}^{2}(t), q_{2}^{2}(t), q_{3}^{2}(t) \& q_{4}^{2}(t)$ are combined in a specific manner to get $\sin ^{2}\left(\delta_{2}(\mathrm{t})\right), \cos ^{2}\left(\delta_{1}(\mathrm{t})\right), \sin ^{2}\left(\lambda_{2}(\mathrm{t})\right)$ $\& \cos ^{2}\left(\lambda_{1}(\mathrm{t})\right)$ signals. In Figure 4 , the first and last branches are configured to detect signal $\cos ^{2}\left(\lambda_{1}(\mathrm{t})\right)$ whereas the middle two branches are configured to detect signal $\sin ^{2}\left(\lambda_{2}(t)\right)$.

\footnotetext{
Corresponding author: qasimumar.khan@ceme.nust.edu.pk
} 


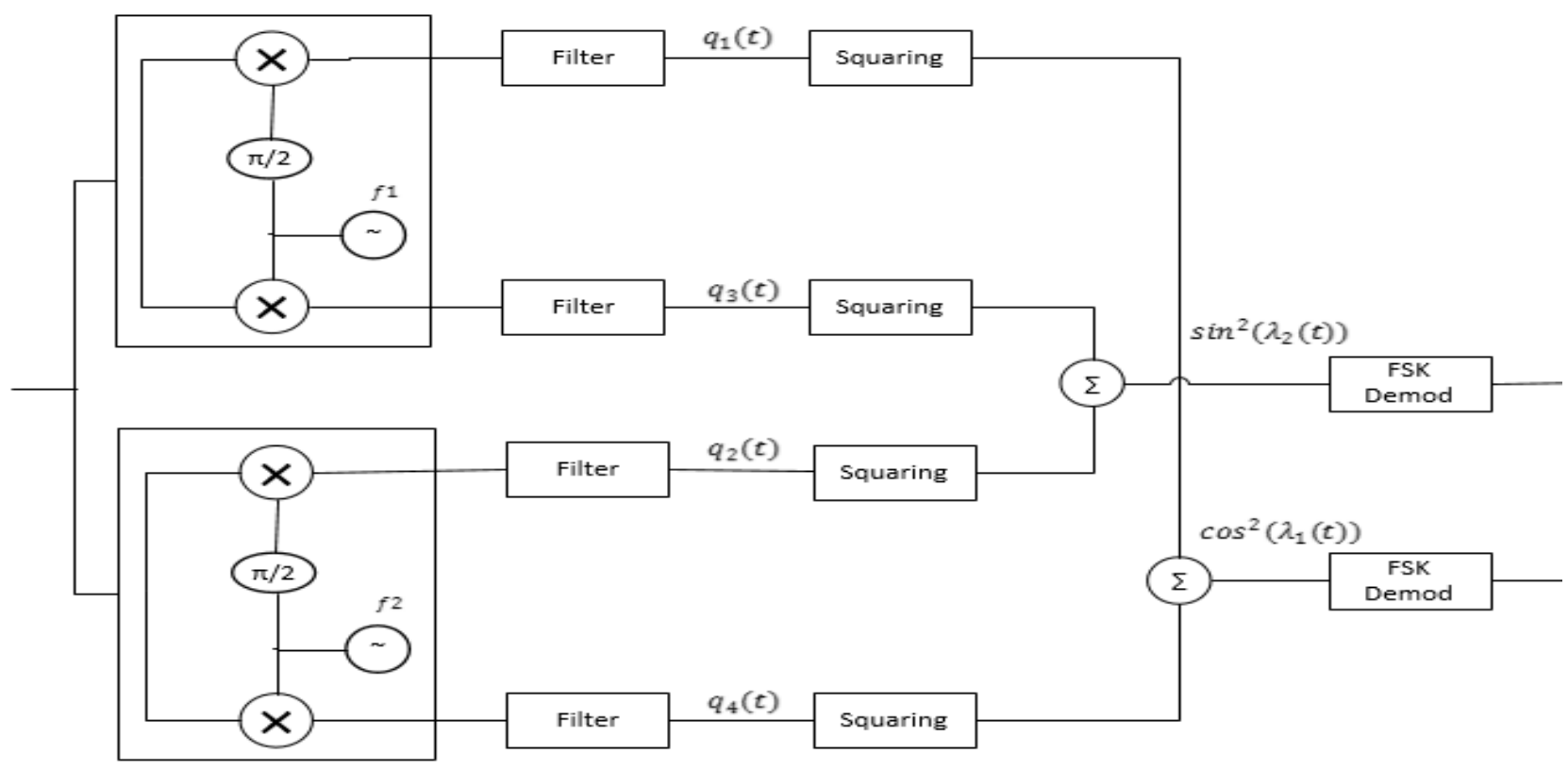

Fig. 4. Basic Two-carrier Digitized Quaternion Demodulator

In Figure 4 the summation of $q_{1}^{2}(t) \& q_{4}^{2}(t)$ gives the square of first symbol $\cos ^{2}\left(\lambda_{1}(t)\right)$ shown in Figure $2(a)$. Mathematically;

$$
\begin{aligned}
q_{1}^{2}(t)+q_{4}^{2}(t) & =\cos ^{2}\left(\lambda_{1}(t)\right) \cdot \sin ^{2}\left(\delta_{2}(t)\right)+\cos ^{2}\left(\lambda_{1}(t)\right) \cdot \cos ^{2}\left(\delta_{1}(t)\right) \\
& =\cos ^{2}\left(\lambda_{1}(t)\right)\left[\sin ^{2}\left(\delta_{2}(t)\right)+\cos ^{2}\left(\delta_{l}(t)\right)\right]
\end{aligned}
$$

Using bandpass filter to get the desired part;

$$
q_{1}^{2}(t)+q_{4}^{2}(t)=\cos ^{2}\left(\lambda_{1}(t)\right)
$$

Similarly the summation of $q_{2}^{2}(t) \& q_{3}^{2}(t)$ gives the square of second symbol $\sin ^{2}\left(\lambda_{2}(t)\right)$, shown in Figure 2(a). Mathematically;

$$
q_{2}^{2}(t)+q_{3}^{2}(t)=\sin ^{2}\left(\lambda_{2}(t)\right)
$$

In Figure 5 the upper two branches are configured to detect signal $\sin ^{2}\left(\delta_{2}(\mathrm{t})\right)$ whereas the lower two branches are configured to detect signal $\cos ^{2}\left(\delta_{1}(t)\right)$. The summation of $\mathrm{q}_{1}^{2}(\mathrm{t}) \& \mathrm{q}_{3}^{2}(\mathrm{t})$ gives the square of forth symbol $\sin ^{2}\left(\delta_{2}(t)\right)$ shown in Figure 2(b). Mathematically; $q_{1}^{2}(t)+q_{3}^{2}(t)=\cos ^{2}\left(\lambda_{1}(t)\right) \cdot \sin ^{2}\left(\delta_{2}(t)\right)+\sin ^{2}\left(\lambda_{2}(t)\right) \cdot \sin ^{2}\left(\delta_{2}(t)\right)$ $=\sin ^{2}\left(\delta_{2}(t)\right)\left[\cos ^{2}\left(\lambda_{1}(t)\right)+\sin ^{2}\left(\lambda_{2}(t)\right)\right]$

Using bandpass filter to get the desired part;

$$
q_{1}^{2}(t)+q_{3}^{2}(t)=\sin ^{2}\left(\delta_{2}(t)\right)
$$

In Figure 5 the summation of squaring of $\mathrm{q}_{2}{ }^{2}(\mathrm{t}) \& \mathrm{q}_{4}{ }^{2}(\mathrm{t})$ gives the square of third symbol $\cos ^{2}\left(\delta_{1}(\mathrm{t})\right)$ shown in Figure 2(b). Mathematically;

$$
q_{2}^{2}(t)+q_{4}^{2}(t)=\cos ^{2}\left(\delta_{I}(t)\right)
$$

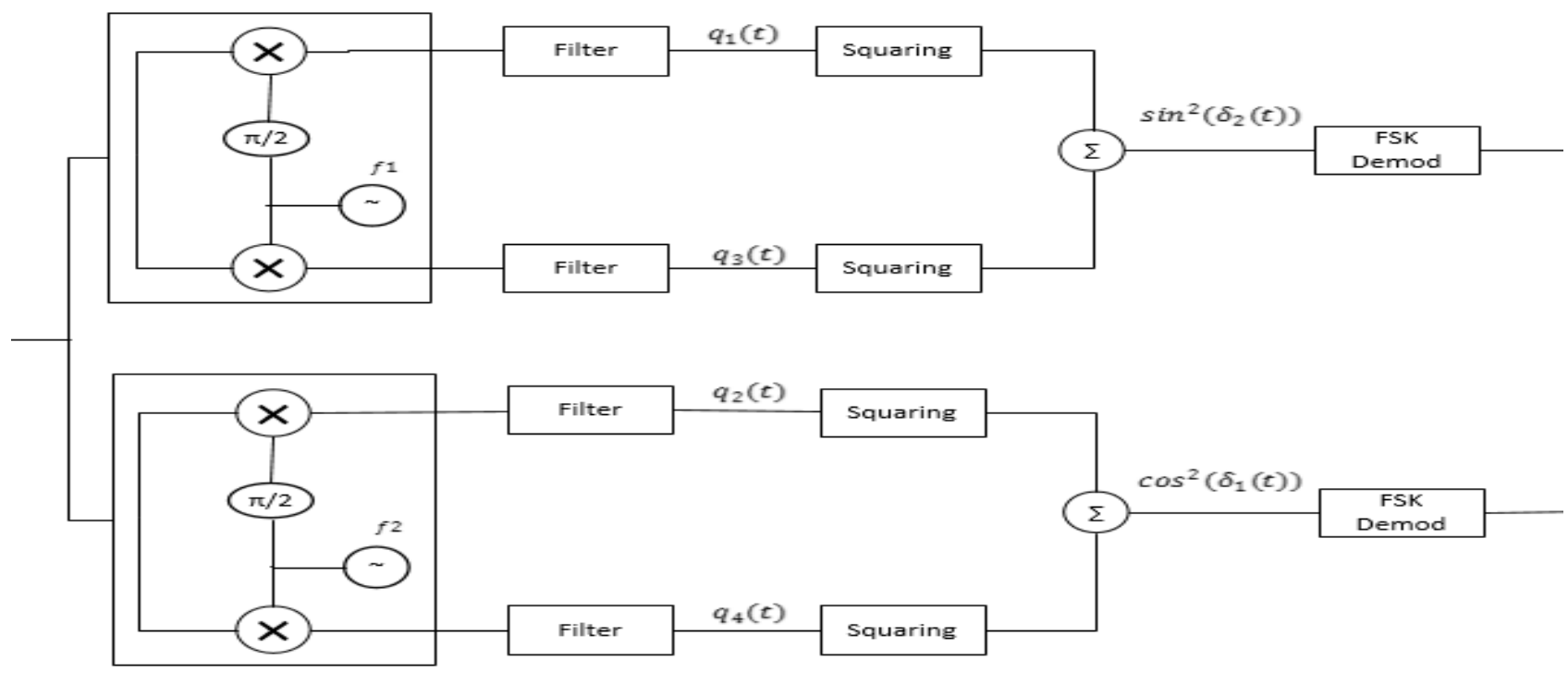

Fig. 5. Basic Two-carrier Digitized Quaternion Demodulator

\footnotetext{
Corresponding author: qasimumar.khan@ceme.nust.edu.pk
} 
These squared signals $\cos ^{2}\left(\lambda_{1}(\mathrm{t})\right), \sin ^{2}\left(\lambda_{2}(\mathrm{t})\right), \sin ^{2}\left(\delta_{2}(\mathrm{t})\right)$ $\& \cos ^{2}\left(\delta_{1}(\mathrm{t})\right) \quad$ undertake final demodulation process which is achieved with FSK demodulation. FSK modulators and demodulators are used to provide good noise immunity properties.

Following are the steps of digitized quaternion modulation scheme.

\section{At the transmitter}

Step 1. Generate random data source

Step 2. Apply FSK modulation with quadrature carriers

Step 3. Generate quaternion coordinates

Step 4. Multiplexing with quadrature carriers

Step 5. Pass the signal through AWGN channel.

\section{At the receiver}

Step 6. Carrier demodulation to get quaternion coordinates.

Step 7. Squaring of quaternion coordinates.

Step 8. Squared quaternions are combined in a specific manner to get squared signals.

Step 9. FSK demodulation

$$
\begin{aligned}
& q_{1}^{2}(t)+q_{4}^{2}(t)=\cos ^{2}\left(\lambda_{1}(t)\right) \\
& q_{2}^{2}(t)+q_{3}^{2}(t)=\sin ^{2}\left(\lambda_{2}(t)\right) \\
& q_{1}^{2}(t)+q_{3}^{2}(t)=\sin ^{2}\left(\delta_{2}(t)\right) \\
& q_{2}^{2}(t)+q_{4}^{2}(t)=\cos ^{2}\left(\delta_{1}(t)\right)
\end{aligned}
$$

Step 10. Calculate the number of errors.

\section{Simulation Results}

The performance of digitized Quaternion Modulation in Additive White Gaussian Noise and its comparison with various modulation schemes is shown in Figure 6. From the bit error rate performance of QPSK, QFSK, QAM and digital Quaternion modulation in AWGN, we found that QPSK and QAM behave more or less the similar; FSK shows worst performance whereas digitized quaternion modulation scheme can more effectively mitigate interference and enhances the system performance.

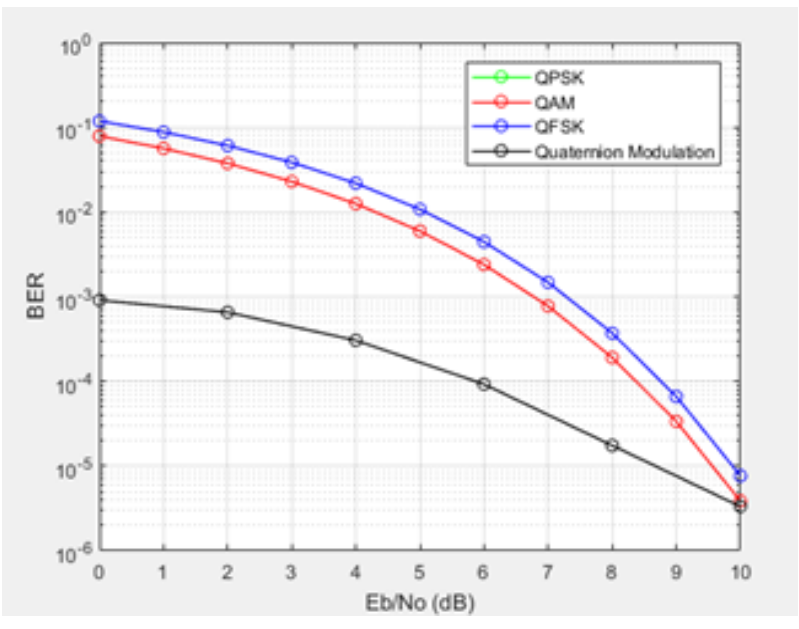

Fig. 6. BER Comparison of QPSK, QFSK, QAM \& Digitized Quaternion Modulation in AWGN.

\footnotetext{
Corresponding author: qasimumar.khan@ceme.nust.edu.pk
}

\section{Conclusion}

This paper shows a digitized quaternion scheme based on complex numbers. A quaternion is composed of four components, one real and three imaginary components. Using FSK systems, the four components are generated and transmitted. The proposed scheme performs better than QPSK, QFSK \& QAM in AWGN channel in terms of BER. This scheme can be used in applications where bandwidth is not an issue i.e. space communication and Global Positioning System (GPS) as these applications need higher precision in reception of data.

\section{References}

1. J. Tewari, H.M. Singh, IJIRCCE, Performance Comparison of Digital Modulation Techniques used in Wireless Communication System, 4,7 (2016).

2. Babu, A Sudhir and Rao, Dr KV Sambasiva, IJCA, Evaluation of BER for AWGN, Rayleigh and Rician fading channels under various modulation schemes, 26, 23 (2011).

3. Sharma, V., Shrivastav, A., Jain, A., \& Panday, A. IJERA, BER performance of OFDM-BPSK,-QPSK,QAM over AWGN channel using forward Error correcting code, 2 ,1619 (2012).

4. Gupta, B., Gupta, G., \& Saini, D. S., ICECT , BER performance improvement in OFDM system with ZFE and MMSE equalizers, 6, 193 (2011).

5. Beebe, Nelson HF, A Bibliography of Publications about the Mathematica Symbolic Algebra Language, (2019).

6. Rosenfeld, Boris A, A history of non-Euclidean geometry: evolution of the concept of a geometric space, 12, (2012).

7. Kavan, L., Collins, S., Žára, J., \& O'Sullivan, C., Skinning with dual quaternions, 39 (2007).

8. H. D. Schütte and J. Wenzel, Hypercomplex numbers in digital signal processing," in Proc. IEEE Int. Symp. Circuits Systems, 1557(1990).

9. Miron, S., Le Bihan, N., \& Mars, J. I., IEEE Transactions on Signal Processing, QuaternionMUSIC for vector-sensor array processing., 54, 1218 (2006).

10. Kantor, I. L., Kantor, I. L., \& Solodovnikov, A. S., Hypercomplex numbers, an elementary introduction to algebras, (1989).

11. J. P. Ward, MA, Quaternions and Cayley Numbers: Algebra and Applications, ser, 403 (1997).

12. Oliveira, H. M., Oliveira, D. R., \& Souza, R. M., XXIX Simpósio Brasileiro de TelecomunicaçõesSBrT, A unit quaternion-based spherical trigonometry and a new two-carrier phasequadrature quaternion modulation system. 05 (2011). 